\title{
Membrane tension regulates syndecan-1 expression through actin remodelling
}

Weiqi Li and Wen Wang*

Institute of Bioengineering and School of Engineering and Materials Science, Queen Mary

University of London, London E1 4NS, UK

\section{* Correspondence}

Professor Wen Wang

Email: wen.wang@qmul.ac.uk

Telephone: +442078823031

Dr Weiqi Li

Email:wei-qi.li@qmul.ac.uk

Telephone: +44 2078827821 


\begin{abstract}
The endothelial glycocalyx, located at the interface of vascular lumen, is a carbohydrate-rich complex that controls vascular functions such as solute permeation, neutrophil extravasation and mechanotransduction. It anchors to the cell membrane through core proteins, e.g. syndecan-1, which couple to the actin cytoskeleton. Membrane tension plays an important role in the reorganisation of membrane-bound proteins, however, little is known on the effect of the membrane tension on the various components of the glycocalyx. In the current study, we use hypo-osmotic stress to investigate the effect of the membrane tension on syndecan-1 expression. Our results show that following 20 min exposure to hypo-osmotic medium, the expression of syndecan-1 in the endothelial glycocalyx layer is reduced to $84.7 \pm 3.6 \%(255 \mathrm{mOsm})$ and $64.7 \pm 2.1 \%(167 \mathrm{mOsm})$. This reduction, however, is transient and partial recovery is observed at the end of $2 \mathrm{~h}$ exposure to the hypo-osmotic medium. The transient reduction of syndecan-1 is associated with depolymerisation of the actin cytoskeleton. Further examination of the effect of actin manipulation reveals that actin depolymerisation by cytochalasin $\mathrm{D}$ results in sustained syndecan-1 reduction. In contrast, stabilising actin using jasplakinolide abolishes the transient reduction of syndecan-1 completely. Taken together, we demonstrate, for the first time, that membrane tension plays an important role in the regulation of syndecan- 1 expression and this effect is mediated by the reorganisation of the actin cytoskeleton. Findings in this study suggest a new venue of research on the protective role of the glycocalyx in vascular pathophysiology and diseases.
\end{abstract}

\title{
Keywords
}

Endothelial glycocalyx; cytoskeleton; hypo-osmotic stress; vascular biology 


\section{Introduction}

Plasma membrane tension, originally known as a passive participant in cell deformation, now becomes more recognised as an active, physical regulator. It is involved in cell spreading, migration and division, which help to maintain the shape and polarity of a cell $[1,2]$. It also coordinates with membrane trafficking, thus generating a feedback to the membrane reservoir where the tension needs to be further adjusted $[3,4]$. Although membrane tension generally affects membrane proteins, its role in adaptation of the endothelial glycocalyx remains unclear.

The endothelial glycocalyx, a glycosaminoglycans (GAGs)-rich complex, mounts on the endothelial cell membrane through a covalent bond with core proteins. The glycocalyx lines on the luminal surface of vasculatures and contributes significantly to the vascular pathophysiology [5]. It is believed that this is fulfilled by disrupting its molecular sieve (to enhance solute permeation), compromising barrier function (to facilitate leukocyte extravasation) and inhibiting nitric oxide production (to dysregulate the vasodilatory activity of blood vessels) [6]. Currently, loss of the endothelial glycocalyx has been closely linked with the development and progression of many diseases, including, but not limited to, atherosclerosis, nephrotic syndrome and sepsis [7-9].

Syndecan-1 is one of the most studied core proteins in the endothelial glycocalyx. The syndecan-1 core protein is secured by the transmembrane spanning domain and contains multiple binding sites for GAGs on the ectodomain, with 1 or 2 chondroitin sulphate chains (CS) for every 4 heparan sulphate (HS) [10]. It also occurs in the form of dimers or oligomers which enable the activation of receptor kinases [11]. It has been reported that inflammatory factors, such as thrombin and matrix metalloproteinases, can trigger proteolytic cleavages on 
the ectodomain of syndecan-1, which in turn leads to a significant loss of HS and CS [12, 13]. On the opposite, inhibiting proteolytic activities of these factors, for example, using sphingosine-1-phosphate, is able to retain the integrity of syndecan-1 as well as its conjugated GAGs $[14,15]$. Syndecan-1 also connects closely with the actin cytoskeleton inside the cytoplasm, making it as an ideal adaptor to convert extracellular mechanical signals to biophysical/chemical reactions through an entire cell. Tarbell and co-workers found that laminar shear flow induced actin reorganisation and subsequent cell alignment through HS. This was particularly achieved by syndecan-1 rather than other core proteins such as glypican$1[16]$. Other study demonstrated that loss of syndecan-1 caused endothelial cells shifting to an inflammatory phenotype in response to atheroprotective flow [17], leading to an abnormally enhanced cytokine expression and leukocyte recruitment. Owing to its spatial distribution and essential role in the endothelial glycocalyx, deciphering the adaptation of syndecan-1 to membrane tension is of great interest and may offer an alternative way to deepen the understanding in the regulation of the endothelial glycocalyx.

In the current study, we employ hypo-osmotic stress to manipulate the cell membrane tension. We observe syndecan-1 expression under both acute (i.e. $20 \mathrm{~min}$ ) and chronic (i.e. 2 h) hypoosmotic conditions. Next, we visualise and quantify the actin cytoskeleton in order to establish a correlation between cell membrane syndecan-1 expression and cytoskeletal reorganisation. This is accompanied with measurement of cellular stiffness and junctional integrity, which enable us to confirm the participation of the actin. Furthermore, we use chemicals to manipulate cytoskeletal actin remodelling under hypo-osmotic stress to establish the mechanism through which membrane tension regulates the expression of syndecan-1. 


\section{Materials and methods}

2.1 Cell culture and hypo-osmotic stress

Human umbilical vein endothelial cells (HUVECs, Lonza, Slough, UK) were cultured until confluence using medium 199 (Fisher scientific, Loughborough, UK) supplemented with foetal bovine serum (10\%, Labtech, Sussex, UK), $\beta$-endothelial cell growth factor $(2.5 \mathrm{ng} / \mathrm{ml}$, Sigma, Dorset, UK), bovine neural extract ( $7.5 \mu \mathrm{g} / \mathrm{ml}$, Sigma), heparin (2.5 U/ml, Sigma), penicillin (1 U/ml, Sigma) and streptomycin (1 $\mu \mathrm{g} / \mathrm{ml}$, Sigma). Membrane tension was altered using hypo-osmotic stress as those previously described [18]. In brief, the abovementioned medium $(313 \mathrm{mOsm} / \mathrm{kg})$ without serum was diluted in ultra-pure water according to a ratio of 9:1, 4:1 and 1:1, which would result in changes of medium osmolality to $283 \mathrm{mOsm} / \mathrm{kg}, 255 \mathrm{mOsm} / \mathrm{kg}$ and $167 \mathrm{mOsm} / \mathrm{kg}$, respectively (Osmometer 3250, Advanced Instruments Ltd, Horsham, UK). The first two values were considered as mild membrane tension alteration, while the last was defined as great enhancement. The $\mathrm{pH}$ values remain unchanged $( \pm 0.1)$ before and after medium modification. HUVECs were exposed to hypo-osmotic medium for a period of $20 \mathrm{~min}$ and $2 \mathrm{~h}$ unless otherwise stated.

\subsection{Actomyosin manipulation}

The role of actin dynamics was investigated using cytochalasin D (Cyto D), blebbistatin (Bleb) and jasplakinolide (Jask), all purchased from Sigma. We incubated HUVECs with optimised concentration of those drugs (diluted in pure medium) for $1 \mathrm{~h}$ prior to hypo-osmotic stress. These were subsequently replaced with the same concentration of drugs which were diluted in hypo-osmotic medium, thus maintaining cells in their depolymerised or hyper-polymerised state before and throughout the experiment [19]. The degree of actin (de)polymerisation was 
assessed in the form of stress fibres and cortical actin, both of which would be described in session 2.4 .

\subsection{Immunofluorescence staining}

Immediately after hypo-osmotic stress, HUVECs were fixed in paraformaldehyde (4 \% in PBS, Sigma) for $10 \mathrm{~min}$. To label syndecan-1 exclusively bound on the cell surface, fixed cells without permeabilisation were pre-blocked with BSA (2\% in PBS, Sigma) for $30 \mathrm{~min}$ and subsequently incubated with mouse-anti-human syndecan-1 (1:75, BA-38, Abcam, Cambridge, UK) in BSA for $2 \mathrm{~h}$. The selected antibody binds to the extracellular domain of human syndecan-1 within an amino acid range of 23-254. This was subsequently visualised by Atto 488 goat-anti-mouse Ig G (1:200, Sigma) and counterstained with DAPI (1:500, Sigma) in

BSA for an additional $1 \mathrm{~h}$. For visualisation of the actin cytoskeleton, fixed cells were permeabilised with PBT (0.25 \% Triton X-100 in PBS, Sigma) for 10 min, blocked with BST (2\% BSA in $0.1 \%$ PBT) for 30 min and then labelled with Atto 565 or Atto 647 phalloidin (1:200, Sigma) and DAPI simultaneously in BST for $1 \mathrm{~h}$. To examine other cytoskeletal structures such as microtubule and spectrin, permeabilised cells were blocked with BST for 30 min and then incubated with mouse-anti-human $\alpha$-tubulin (1:200, TU-02) and spectrin $\alpha$ II $(1: 100, C 3)$ in BST, respectively. Both antibodies were purchased from Santa Cruz (Insight Biotechnology Ltd, Middlesex, UK). Those cells were then incubated with Atto 488 or Atto 546 goat-anti-mouse Ig G (1:200, Sigma) concurrent with phalloidin and DAPI in BST for an additional $1 \mathrm{~h}$. All the labelled samples were mounted in ProLong ${ }^{\circledR}$ Gold anti-fade reagent (Fisher scientific) and scanned by confocal microscopy within one week. 
2.4 Image acquisition and analysis

Samples were scanned using a Zeiss 710 confocal laser scanning microscope with a Zeiss PlanAPOCHROMAT 63×/1.4 Oil DIC (Cambridge, UK). 8-bit images were acquired from basal cell surface to apical cell surface at an interval of $0.3 \mu \mathrm{m}$. Laser power, gain and offset value were optimised based on pseudo-colour to achieve optimal brightness and to avoid photobleaching. In some cases, sequential scanning mode was employed to prevent crosstalk between two different fluorophores. Confocal z-stack images were shown using maximumintensity projection in Fiji (NIH) [20]. Syndecan-1 expression was determined by reading thresholded fluorescence intensity on each cell and presented as percentage normalised to the parallel control, i.e. pure medium $[19,21]$. To quantify the actin cytoskeleton, stacked images were separated according to the curved membrane. Sections from the start of curvature were projected again to obtain actin cortex image. Measurement of the cortical intensity was performed in the same region as cell nucleus on each cell [22]. The intensity was then normalised with respect to the parallel control. Those sections beyond the curvature were restacked, cropped to individual cell size and then imported into FilaQuant for basal filament counting $[19,23]$.

\subsection{Measurement of mechanical properties}

Cell mechanics were examined using JPK NanoWizard 4 system (Bruker, Coventry, UK) mount on an inverted microscope (Axio Observer Z1, Zeiss). Cantilevers (OMCL-800PSA-1, Olympus, Essex, UK) were calibrated by first measuring the sensitivity against the stiff polystyrene substrate, and then fitting the resonance peak in the thermal noise spectrum to determine the spring constant, i.e. $0.05 \mathrm{~N} / \mathrm{m}$ [24]. A ROI of $64 \mu \mathrm{m} \times 64 \mu \mathrm{m}$ was selected to cover an entire cell. Indentation with a set point of $2 \mathrm{nN}$ was performed in a format of $32 \times 32$ 
using advanced quantitative imaging mode. Loading and unloading rate were set to $50 \mu \mathrm{m} / \mathrm{s}$, which allowed us to achieve fast data acquisition without compromising the image resolution. Data collection were carried out at $37^{\circ} \mathrm{C}$ in serum-free, hypo-osmotic medium and completed within $30 \mathrm{~min}$. All the data at each group were randomly distributed and no downward/upward trend was observed during this period of time. Force-indentation curves over the region of cytoplasm were extracted and processed in JPK data processing software. Following a number of steps including background subtraction, height correction, contact point determination and fitting with a modified hertz model (Eq.1), indentation depth $(\delta)$ over $300 \mathrm{~nm}$ was used to determine global cell stiffness (E, young's modulus, $\mathrm{kPa}$ ), whilst range within the first $100 \mathrm{~nm}$ was defined as cortical stiffness [22, 25].

$$
F=0.7453 \frac{E}{1-v^{2}} \delta^{2} \tan \alpha
$$

Where the poisson's ratio, $v$, is assumed to be 0.5 and the half face angle of a four-sided pyramid cantilever, $\alpha$, is set to be $41^{\circ}$ according to scanning electronic microscope images. Cell height, as calculated from a line drawn across basal cell membrane to the apex, was also given at the end of processing.

\subsection{Determination of cell permeability}

HUVECs on Transwell inserts (Pore size $=3 \mu \mathrm{m}$, Corning, Sigma) were pre-incubated with hypo-osmotic medium for $20 \mathrm{~min}$ and $2 \mathrm{~h}$. It was followed by a replacement of FITC-dextran $40 \mathrm{kDa}$ (Sigma) which was prepared in respective hypo-osmotic medium. The osmotic pressure is always equalised at both sides of the insert throughout the assay. Medium in the bottom compartment were collected after 5 min incubation and measured using fluorescence plate reader with a gain value of 500 (FLUOstar OPTIMA, BMG Labtech, Aylesbury, UK). All the 
readings are normalised to values obtained from 20min, $283 \mathrm{mOsm}$, and presented as relative permeation.

\subsection{Statistical analysis}

Data was presented as mean \pm standard error and statistical analysis was performed in SPSS 24 using one way or two-way ANOVA with Tukey's HSD for multiple means comparison. Difference was considered significant if $P<0.05$.

\section{Results}

3.1 Transient increase in the membrane tension reduces syndecan-1 expression.

The glycocalyx anchors to the cell membrane through core proteins such as syndecan- 1 . Those proteins serve as an adaptor that converts mechanical signals to biochemical and biophysical reactions within a cell. In this study the effect of membrane tension on syndecan-1 expression was investigated using hypo-osmotic stress. In order to find out the gradient dependence, we incubated HUVECs in hypo-osmotic medium with decreasing osmolality. By using atomic force microscopy (AFM), we found that cell height changed from $3.1 \pm 0.2 \mu \mathrm{m}(313 \mathrm{mOsm})$ to $2.9 \pm 0.1 \mu \mathrm{m}(283 \mathrm{mOsm}), 3.3 \pm 0.1 \mu \mathrm{m}(255 \mathrm{mOsm})$ and $4.0 \pm 0.2 \mu \mathrm{m}(167 \mathrm{mOsm})$ as early as 20 min following the onset of hypo-osmotic stress (Fig. 1c). At $2 \mathrm{~h}$, there was no further increase in the cell height, indicating a steady state under sustained hypo-osmotic stress. These results showed cell swelling and associated increase in the membrane tension without bursting of the cell membrane. Next we performed immunofluorescence to visualise syndecan-1 on the cell surface. Confocal images revealed that syndecan-1 staining became less intensive at 20 min when the medium osmolality was reduced. However, following $2 \mathrm{~h}$ stimulation, the 
fluorescence intensity of syndecan-1 was recovered (Fig 1.a). Normalised data confirmed the expression of syndecan-1 was significantly declined from $103.8 \pm 2.9 \%$ (283 mOsm) to $84.7 \pm 3.6 \%(255 \mathrm{mOsm})$ and $64.7 \pm 2.1 \%(167 \mathrm{mOsm})$ at $20 \mathrm{~min}$. This was followed by recoveries to $98.8 \pm 4.6 \%(255 \mathrm{mOsm})$ and $85.9 \pm 3.2 \%(167 \mathrm{mOsm})$ at the end of $2 \mathrm{~h}$, indicating restoration of syndecan-1 (Fig. 1b). Changing the medium osmolality also alters ion and glucose concentrations. In order to exclude the influence of ions and glucose in the osmoregulation, the hypo-osmotic medium of 167 mOsm was compensated with $150 \mathrm{mM}$ mannitol to generate an osmolality of $320 \mathrm{mOsm}$, which is similar to the medium (313 mOsm). Under such condition, concentrations of ion and glucose remained the same as in the diluted medium (167 mOsm). Our results showed that the expression of syndecan-1 at 20 min and $2 \mathrm{~h}$ was sustained at $98.9 \pm 1.6 \%$ and $106.2 \pm 2.1 \%$, respectively (Fig. 1b), suggesting that is the decreased osmolality that leads to the reduction of syndecan-1. On the other hand, we had the concentrations of individual ions and glucose corrected in the hypo-osmotic medium (Fig. S5). The still detectable reduction confirmed the fact that ions and glucose do not interfere with the osmoregulation of syndecan-1.

3.2 The transient reduction of syndecan-1 is associated with disrupted actin polymerisation.

Membrane tension coordinates with the actin cytoskeleton in the regulation of membrane events. To explore the mechanism underlying the transient reduction of syndecan-1, we examined its correlation to the actin cytoskeleton organisation. As shown in Fig. 2a, two phases of actin reorganisation were observed in confocal images at 167 mOsm. Complete actin disruption occurred following 20 min stimulation, whereas repolymerisation, albeit not to a fully polymerised level, was found at $2 \mathrm{~h}$. This response was inhibited if the hypo-osmotic medium was tuned to iso-osmotic by mannitol (Fig. S6), indicating the actin depolymerisation 
is also attributed to the change of medium osmolality. Actin cytoskeleton has a great impact on cell mechanics which is coupled with the membrane tension. To verify the occurrence of the two-phase response, cell stiffness and cortical stiffness, both of which are dominated by the actin cytoskeleton, were measured using AFM indentation. Young's modulus significantly decreased from $1.4 \pm 0.1 \mathrm{kPa}(283 \mathrm{mOsm})$ to $0.9 \pm 0.1 \mathrm{kPa}(167 \mathrm{mOsm})$ at $20 \mathrm{~min}$ (Fig. 2b). This is in accordance to the loss of actin as shown in the confocal images. Although the value of the modulus recovered to $1.2 \pm 0.1 \mathrm{kPa}(167 \mathrm{mOsm})$ at $2 \mathrm{~h}$, the magnitude was still lower than the control, indicating that only a partial re-polymerisation is achieved at $2 \mathrm{~h}$ (Fig 2.b). Similarly, the cortical stiffness dropped from $5.6 \pm 0.3 \mathrm{kPa}(283 \mathrm{mOsm})$ to $2.8 \pm 0.1 \mathrm{kPa}(167 \mathrm{mOsm})$ at 20 min and followed with a recovery to $3.4 \pm 0.2 \mathrm{kPa}$ at the end of $2 \mathrm{~h}$ (Fig. 2c). Owing to the remedy effect of mannitol, the reduction in cellular stiffness was not observed (Fig. S1). In addition to the actin, microtubule and spectrin were investigated to give a glimpse on the contribution from other cytoskeletal systems. The polymerisation of microtubule and spectrin seems sustained irrespective of the extent of hypo-osmotic stress and the exposure time (Fig. 2a), even though the intensity of spectrin was declined at 167 mOsm (Fig. S2), probably due to the loss of interconnection between the actin and the spectrin. Furthermore, the hypo-osmotic stress at $167 \mathrm{mOsm}$ also led to an increased permeation of FITC-dextran $40 \mathrm{kDa}$ across endothelial cell monolayer (Fig. 2d). All these results demonstrated that the transient reduction of syndecan-1 is associated with temporary actin depolymerisation.

3.3 The transient actin depolymerisation was restored in the presence of jasplakinolide.

Having demonstrated the correlation between syndecan-1 expression and actin reorganisation, the role of actin dynamics in the transient reduction of syndecan-1 was investigated, using cytochalasin D (Cyto D, $100 \mathrm{nM}$ ) and jasplakinolide (Jask, $15 \mathrm{nM})$. Those drug effects initiated 
at $1 \mathrm{~h}$ before the onset of hypo-osmotic stress and lasted throughout the stimulation. As expected, the actin cytoskeleton was completely depolymerised by Cyto D (Fig. S3). This was independent of the extent of hypo-osmotic stress and the exposure time. On the other hand, in the presence of Jask, the actin polymerisation was clearly preserved against 167 mOsm hypoosmotic stress (Fig. S3). The restoration effect was compromised to some extent at the end of $2 \mathrm{~h}$, likely due to an over-incubation of drug. In addition, actin polymerisation is coordinated by the activity of non-muscle myosin II. We also conducted experiment using blebbistatin (Bleb, $12.5 \mu \mathrm{M}$ ) to assess the involvement of actomyosin contractility. Surprisingly, the cytoskeleton remained polymerised in response to $283 \mathrm{mOsm}$ hypo-osmotic stress at $20 \mathrm{~min}$ (Fig. S3a). The failure to facilitate actin disruption here suggested that the non-muscle myosin II may not be involved in the tension-induced actin disruption. After $2 \mathrm{~h}$ stimulation, the actin failed to reorganise, which thereafter led to varying degrees of cell shrinkage (Fig. S3b). The stress induced by the hypo-osmotic medium is distributed throughout the cell and not just concentrated in the cortex or fibres. Those basal fibres and the cortical actin provide joint support to the membrane proteins on the apical surface. It was shown that without any cytoskeletal drug, basal filament number significantly decreased from $37.7 \pm 2.6$ (283 mOsm) to $1.0 \pm 0.5(167 \mathrm{mOsm})$ at $20 \mathrm{~min}$ (Fig. 3a). This was confirmed according to the usage of Cyto $\mathrm{D}$, where filament number under both conditions dropped to 1.0 or less. The loss of filament number at $20 \mathrm{~min}$ was not affected by Bleb at all but could be reversed to $42.7 \pm 2.0$ (167 mOsm) in the presence of Jask (Fig. 3a). Similarly, in the absence of cytoskeletal drugs, there was a remarkable decline in cortical intensity, from $100 \pm 5.5 \%$ (283 mOsm) to $66.5 \pm 5.2 \%$ (167 mOsm). The intensity was, however, retrieved to $102.1 \pm 6.2 \%$ (167 mOsm) when Jask was introduced (Fig. 3b). The changes in filament number and cortical intensity at $2 \mathrm{~h}$ remained similar to $20 \mathrm{~min}$, except those with the treatment of Bleb (Fig. 3d). The cortical intensity there was surprisingly enhanced, which was likely due to cell shrinkage with a resulting aggregation 
of cortical actin. It was also worth mentioning that cortical intensity, unlike filament number, was always detachable with or without depolymerisation, implying that actin cortex is less vulnerable than stress fibres in response to cytoskeletal drugs.

3.4 The transient reduction of syndecan-1 is counteracted by the stabilization of actin polymerisation.

At the end of this study, the expression of syndecan-1 was assessed using hypo-osmotic stress in combination with actin manipulation. In the presence of $\mathrm{CD}$, a reduction of syndecan- 1 at 20 min was observable $(73.4 \pm 5.1 \%)$ even if the medium osmolality was slightly reduced (283 mOsm). This was also sustained at the end of $2 \mathrm{~h}$ exposure, where the expression was at a reduced level of $76.6 \pm 3.5 \%$ (167 $\mathrm{mOsm})$. These results demonstrated that actin polymerisation is essential in terms of maintaining the coverage of syndecan-1. On the other hand, applying Bleb neither promoted further reduction at $20 \mathrm{~min}(68.8 \pm 2.9 \%)$, nor inhibited recovery of syndecan-1 at $2 \mathrm{~h}(82.5 \pm 5.3 \%)$, suggesting that actomyosin contractility does not contribute to the preservation of syndecan-1. On the contrary, through stabilizing the actin cytoskeleton with Jask, the transient reduction of syndecan-1 was completely abolished. The expression of syndecan-1 at 20 min was as high as those without hypo-osmotic stress $(99.6 \pm 4.2 \%)$, and the preservation was even prolonged up to $2 \mathrm{~h}(97.4 \pm 5.6 \%)$. The finding once again supported our hypothesis that membrane tension alters the expression of sydecan-1 through adapting actin polymerisation.

\section{Discussion}

Hypo-osmotic stress is a common approach to increase membrane tension [26, 27]. Under such circumstance, elevation of membrane tension is accompanied with an increase in cell volume, 
which enables us to evaluate the change of membrane tension using simple parameters such as cell height [18]. Here we show that cell height increases with the reduction of medium osmolality, with the plateau reached at $167 \mathrm{mOsm}$. The increase in cell volume is not followed by a regulated volume decrease (VRD) which is normally found on in situ and freshly isolated chondrocytes [28]. Many types of cells response to hypo-osmotic stress rapidly through swelling but the subsequent VRD extends from minutes to several hours, depending on the compensatory mechanism, e.g. altered transmembrane electrical potential difference and the efflux of $\mathrm{K}^{+}$[29]. The discrepancy between these two cell types is likely attributed to the respective growth environment, where endothelial cells are adherent, lining on top of extracellular matrix, whilst chondrocytes are embedded, with a high susceptibility as well as adaptability to the alteration in the extracellular environment. We limit hypo-osmotic stress to $2 \mathrm{~h}$, as over-stimulation can easily compromise cell viability and cell attachment, both of which affect cell behaviour accordingly. It should be pointed out that our experimental conditions are not completely physiologically relevant. The osmolality of the serum ranges from $275 \mathrm{mOsm}$ to $295 \mathrm{mOsm}$ and is tightly regulated $(\leq 10 \%)$ even if suffering from compulsive water drinking [30]. If using such settings in an in vitro study, it would be much harder to demonstrate its effect on changes in the endothelial glycocalyx. The primary goal of the current study is to use hypo-osmotic stress as an approach to manipulate endothelial cell membrane tension and its effect on the glycocalyx component.

A transient increase in the membrane tension, in particular at hypo-osmotic stress of $167 \mathrm{mOsm}$, reduces the syndecan-1 expression in the endothelial glycocalyx. Sustained membrane tension over $2 \mathrm{~h}$ stimulates a reformation of syndecan- 1 to compensate its compromised coverage. The reduction and the subsequent recovery in syndecan-1 are observed to be highly associated with the polymerised state of the actin cytoskeleton. Actin cytoskeleton becomes completely 
depolymerised at $20 \mathrm{~min}$, but partially re-polymerises at $2 \mathrm{~h}$. Indeed, structural coupling between the actin cytoskeleton and the glycocalyx has long been recognised. Michel and coworkers suggested that the glycocalyx chains were linked to the submembranous cytoskeletal scaffold in a hexagonal format [31]. Since then, its role in triggerring actin reorganisation in response to shear flow has been well documented $[16,32,33]$. Recently, a reciprocate effect from the actin cytoskeleton was reported based on a study in our own laboratory. Using wheat germ agglutinin staining, we showed that without actin polymerisation, the glycocalyx was severely disrupted in the presence of laminar shear flow [19]. This was consistent with Zeng et al's study where the actin cytoskeleton provided a physical support to GAGs, especially HS and CS, against shear stress [34]. Interestingly, at $167 \mathrm{mOsm}$ actin depolymerised throughout the cell centroid, the staining around the cell periphery/junctional region remains strong or becomes stronger after the treatment. This condition can be also found when actin is disrupted by an appropriate concentration of cytochalasin D [19]. We believe this suggests a transition that helps maintaining cell attachment as well as the integrity of the cell monolayer. It implies a plausible mechanism that junctional tension may play an important role in the regulation of syndecan-1.

To elucidate the causal role of actin in the tension-induced syndecan- 1 reduction, we use Jask to stabilise the polymerised actin before and during hypo-osmotic stress stimulation. The reduction in syndecan-1 is completely suppressed. This indicates that stabilizing actin polymerisation counteracts an elevation in the membrane tension and subsequently preserves the coverage of syndecan-1 on the cell membrane. Even though there is an elevation in syndecan-1 following $2 \mathrm{~h}$ mild tension alteration $(283 \mathrm{mOsm})$, the difference is not highly significant. The limited up-regulation in syndecan-1 under such condition implies a feedback loop for actin that retains an adequate amount of syndecan-1 on the endothelial cell surface. 
On the other hand, when Cyto D is used to inhibit actin polymerisation, even mild level of alternation in the membrane tension $(283 \mathrm{mOsm})$ results in a significant reduction in the expression of syndecan-1 at 20 min. The recovery of syndecan-1 is slow at the end of $2 \mathrm{~h}$ exposure. This demonstrates that the adaptation of syndecan-1 to membrane tension requires actin and its remodelling. Meanwhile, we notice that the loss of syndecan-1 is incomplete, as other cytoskeletal components may contribute to the integrity of syndecan-1, even if they are insensitive to a change in the membrane tension. In red blood cell, spectrin interconnects with actin providing a cytoskeletal network for the integrity of plasma membrane as well as stabilisation/activation of membrane receptors and transporters [35]. Spectrin is also highly expressed in endothelial cell system and coordinates with actin assembly [36]. We show that the organisation of spectrin is partially disrupted in response to hypo-osmotic stress, probably emerging as an outcome of collateral damage from the actin depolymerisation. Similarly, depolymerisation or reorganisation is not observed in microtubules. These results demonstrate that neither cytoskeletal elements participate in the transient reduction of syndecan-1 but they may provide support to the remainder of the syndecan-1.

Apart from cytoskeleton itself, we investigate the contribution of actomyosin contractility. Blocking non-muscle myosin II using Bleb has limited effect on the transient reduction in syndecan-1 expression. There is no further transient reduction at higher level of membrane tension (i.e. $167 \mathrm{mOsm}$ ). Non-muscle Myosin II relies on actin cytoskeleton to modulate the contraction. The absence of further reduction implies that the inhibitory effect of Bleb is neglected, which may attribute to the fact that actin filaments are already low under such 
condition (167 mOsm). Furthermore, Bleb does not inhibit the restoration of syndecan-1 at 2 $\mathrm{h}$, suggesting that actomyosin contractility contributes little to the preservation of synecan- 1 .

Despite its actin dependence, we have not determined which specific membrane events are actively involved. It has been reported that alteration in membrane tension has the ability to activate proteins embedded within/conjugated on plasma membrane [37]. This includes some matrix metalloproteinases which are supposed to avoid overexpression of certain types of protein on the cell membrane [38]. Since activation of those enzymes are mainly actinindependent, proteolytic shedding of the syndecan-1 is unlikely to be involved in the tensioninduced reduction of syndecan-1. Stretch-activated ion channels are also implicated. The opening/closing can be triggered through membrane curvature remodelling [39], conformational change in the lipid bilayer or deflection on the tethers formed between the intraand extra-cytoplasmic domain and the cytoskeleton and extracellular matrix, respectively [40]. In addition, it may be argued that the substantial reduction of syndecan- 1 is an outcome of an enlarged cell surface area. Given that the lipid bilayer is a conserved structure and difficult to regenerate, the expansion of surface area is probably due to the excess of membrane area stored in protrusions and invaginations such as microvilli and caveolae [41]. This suggests a significant role of membrane topology in the osmoregulation of syndecan-1. Indeed, our findings show that the syndecan- 1 reduction is transient and can be reversed. There must be a storage that compensates the loss of syndecan-1. de novo synthesis comprises transportations through microtubules and actin filaments. Considering the time that the restoration effect takes place, a $2 \mathrm{~h}$ of exposure does not seem to be sufficient for a substantial recovery through de novo formation. Thus de novo synthesis as a required step is also precluded. Recently, membrane trafficking has been theoretically proposed to be responsible for the alteration of the endothelial glycocalyx from static to laminar flow conditions [42]. Actin dynamics interplaying 
with membrane tension orchestrates vesicle transport in and out of cell membrane [43, 44]. These raise another possibility that membrane trafficking may actively participate in the actindependent, transient reduction of syndecan-1. Ongoing research will look into vesical trafficking such as endosomal recycling and regulated exocytosis which will offer a signalling pathway underlying such regulation.

Actin depolymerisation followed by re-polymerisation suggests an adaptive cellular process to regain a proper membrane tension. Similarly, the transient reduction and the subsequent partial restoration of syndecan-1 also demonstrate an adaptation. Membrane tension depends on the extracellular environment, especially plasma protein and solute concentrations. Temporary leakage of such substances may induce adaptation to attenuate the loss of syndecan-1 or even ameliorate its coverage, however, significant leakage, for example, under the condition of proteinuria, oedema and even sepsis, will definitely result in an irreversible effect that compromises the syndecan-1 as well as other components of the glycocalyx layer. It is, therefore, important to understand the role of membrane tension in the regulation of the glycocalyx.

\section{Conclusion}

We have shown that increasing membrane tension by hypo-osmotic stress results in a transient reduction of the syndecan-1 expression. We have also identified the transient reduction is associated with actin cytoskeletal depolymerisation, which is further verified by decreased cell/cortical stiffness and disrupted cell-cell adhesion. We have demonstrated that the reduction in syndecan-1 is actin-dependant, where stabilisation of the actin cytoskeleton completely inhibits such phenomenon, whereas depolymerising actin is able to maintain and/or advance it. 
In addition, we have ruled out the contribution from microtubule, spectrin and non-muscle myosin II to the transient reduction of syndecan-1. All these findings support our hypothesis that membrane tension regulates syndecan-1 expression through actin remodelling and demonstrate, for the first time, effects of membrane tension on the regulation of the endothelial glycocalyx.

\section{Contributions}

WL and WW designed the study. WL carried out all experiments, performed data analysis and drafted the manuscript. WW supervised this project, checked results and finalised the manuscript. Both authors gave approval for the publication.

\section{Competing financial interests}

The authors declare no competing financial interests.

\section{Acknowledgement}

This work was supported by The Engineering and Physical Sciences Research Council (EPSRC) [EP/R035571/1].

\section{Reference}

[1] N.C. Gauthier, M.A. Fardin, P. Roca-Cusachs, M.P. Sheetz, Temporary increase in plasma membrane tension coordinates the activation of exocytosis and contraction during cell spreading, Proc Natl Acad Sci U S A, 108 (2011) 14467-14472.

[2] A.D. Lieber, S. Yehudai-Resheff, E.L. Barnhart, J.A. Theriot, K. Keren, Membrane tension in rapidly moving cells is determined by cytoskeletal forces, Current biology : CB, 23 (2013) 1409-1417.

[3] A. Diz-Munoz, D.A. Fletcher, O.D. Weiner, Use the force: membrane tension as an organizer of cell shape and motility, Trends Cell Biol, 23 (2013) 47-53. 
[4] N.C. Gauthier, T.A. Masters, M.P. Sheetz, Mechanical feedback between membrane tension and dynamics, Trends Cell Biol, 22 (2012) 527-535.

[5] B.F. Becker, M. Jacob, S. Leipert, A.H. Salmon, D. Chappell, Degradation of the endothelial glycocalyx in clinical settings: searching for the sheddases, British journal of clinical pharmacology, 80 (2015) 389-402.

[6] S. Reitsma, D.W. Slaaf, H. Vink, M.A. van Zandvoort, M.G. oude Egbrink, The endothelial glycocalyx: composition, functions, and visualization, Pflugers Arch, 454 (2007) 345-359.

[7] A.H. Salmon, J.K. Ferguson, J.L. Burford, H. Gevorgyan, D. Nakano, S.J. Harper, D.O. Bates, J. Peti-Peterdi, Loss of the endothelial glycocalyx links albuminuria and vascular dysfunction, Journal of the American Society of Nephrology : JASN, 23 (2012) 1339-1350.

[8] B.M. van den Berg, J.A. Spaan, T.M. Rolf, H. Vink, Atherogenic region and diet diminish glycocalyx dimension and increase intima-to-media ratios at murine carotid artery bifurcation, Am J Physiol Heart Circ Physiol, 290 (2006) H915-920.

[9] J.A. Zullo, J. Fan, T.T. Azar, W. Yen, M. Zeng, J. Chen, B.B. Ratliff, J. Song, J.M. Tarbell, M.S. Goligorsky, B.M. Fu, Exocytosis of Endothelial Lysosome-Related Organelles Hair-Triggers a Patchy Loss of Glycocalyx at the Onset of Sepsis, Am J Pathol, 186 (2016) 248-258.

[10] A. Rapraeger, M. Jalkanen, E. Endo, J. Koda, M. Bernfield, The cell surface proteoglycan from mouse mammary epithelial cells bears chondroitin sulfate and heparan sulfate glycosaminoglycans, J Biol Chem, 260 (1985) 11046-11052.

[11] D.J. Carey, Syndecans: multifunctional cell-surface co-receptors, The Biochemical journal, 327 ( Pt 1) (1997) 1-16.

[12] A.W. Mulivor, H.H. Lipowsky, Inhibition of glycan shedding and leukocyte-endothelial adhesion in postcapillary venules by suppression of matrixmetalloprotease activity with doxycycline, Microcirculation, 16 (2009) 657-666.

[13] S.V. Subramanian, M.L. Fitzgerald, M. Bernfield, Regulated shedding of syndecan-1 and -4 ectodomains by thrombin and growth factor receptor activation, J Biol Chem, 272 (1997) 14713-14720.

[14] K. Hsia, M.J. Yang, W.M. Chen, C.L. Yao, C.H. Lin, C.C. Loong, Y.L. Huang, Y.T. Lin, A.D. Lander, H. Lee, J.H. Lu, Sphingosine-1-phosphate improves endothelialization with reduction of thrombosis in recellularized human umbilical vein graft by inhibiting syndecan-1 shedding in vitro, Acta biomaterialia, 51 (2017) 341-350.

[15] Y. Zeng, R.H. Adamson, F.R. Curry, J.M. Tarbell, Sphingosine-1-phosphate protects endothelial glycocalyx by inhibiting syndecan-1 shedding, Am J Physiol Heart Circ Physiol, 306 (2014) H363-372.

[16] E.E. Ebong, S.V. Lopez-Quintero, V. Rizzo, D.C. Spray, J.M. Tarbell, Shear-induced endothelial NOS activation and remodeling via heparan sulfate, glypican-1, and syndecan-1, Integr Biol (Camb), 6 (2014) 338-347. 
[17] P.L. Voyvodic, D. Min, R. Liu, E. Williams, V. Chitalia, A.K. Dunn, A.B. Baker, Loss of syndecan-1 induces a pro-inflammatory phenotype in endothelial cells with a dysregulated response to atheroprotective flow, J Biol Chem, 289 (2014) 9547-9559.

[18] A. Pietuch, B.R. Bruckner, A. Janshoff, Membrane tension homeostasis of epithelial cells through surface area regulation in response to osmotic stress, Biochim Biophys Acta, 1833 (2013) 712-722.

[19] W. Li, W. Wang, Structural alteration of the endothelial glycocalyx: contribution of the actin cytoskeleton, Biomech Model Mechanobiol, 17 (2018) 147-158.

[20] J. Schindelin, I. Arganda-Carreras, E. Frise, V. Kaynig, M. Longair, T. Pietzsch, S. Preibisch, C. Rueden, S. Saalfeld, B. Schmid, J.Y. Tinevez, D.J. White, V. Hartenstein, K. Eliceiri, P. Tomancak, A. Cardona, Fiji: an open-source platform for biological-image analysis, Nature methods, 9 (2012) 676-682.

[21] K. Bai, W. Wang, Shear stress-induced redistribution of the glycocalyx on endothelial cells in vitro, Biomech Model Mechanobiol, 13 (2014) 303-311.

[22] V. Prystopiuk, B. Fels, C.S. Simon, I. Liashkovich, D. Pasrednik, C. Kronlage, R. Wedlich-Soldner, H. Oberleithner, J. Fels, A two-phase response of endothelial cells to hydrostatic pressure, J Cell Sci, 131 (2018).

[23] C. Matschegewski, S. Staehlke, H. Birkholz, R. Lange, U. Beck, K. Engel, J.B. Nebe, Automatic Actin Filament Quantification of Osteoblasts and Their Morphometric Analysis on Microtextured Silicon-Titanium Arrays, Materials, 5 (2012) 1176-1195.

[24] K. Bai, W. Wang, Spatio-temporal development of the endothelial glycocalyx layer and its mechanical property in vitro, J R Soc Interface, 9 (2012) 2290-2298.

[25] M.A.A. Ayee, E. LeMaster, T. Teng, J. Lee, I. Levitan, Hypotonic Challenge of Endothelial Cells Increases Membrane Stiffness with No Effect on Tether Force, Biophys J, 114 (2018) 929-938.

[26] A. Diz-Munoz, K. Thurley, S. Chintamen, S.J. Altschuler, L.F. Wu, D.A. Fletcher, O.D. Weiner, Membrane Tension Acts Through PLD2 and mTORC2 to Limit Actin Network Assembly During Neutrophil Migration, PLoS biology, 14 (2016) e1002474.

[27] J.P. Ferguson, S.D. Huber, N.M. Willy, E. Aygun, S. Goker, T. Atabey, C. Kural, Mechanoregulation of clathrin-mediated endocytosis, J Cell Sci, 130 (2017) 3631-3636.

[28] Z. Wang, J. Irianto, S. Kazun, W. Wang, M.M. Knight, The rate of hypo-osmotic challenge influences regulatory volume decrease (RVD) and mechanical properties of articular chondrocytes, Osteoarthritis and cartilage, 23 (2015) 289-299.

[29] J. Grantham, M. Linshaw, The effect of hyponatremia on the regulation of intracellular volume and solute composition, Circ Res, 54 (1984) 483-491.

[30] D. K. Faria, M. Mendes, N. M. Sumita, The measurement of serum osmolality and its application to clinical practice and laboratory: Literature review, 2017. 
[31] J.M. Squire, M. Chew, G. Nneji, C. Neal, J. Barry, C. Michel, Quasi-periodic substructure in the microvessel endothelial glycocalyx: a possible explanation for molecular filtering?, J Struct Biol, 136 (2001) 239-255.

[32] J.J. Moon, M. Matsumoto, S. Patel, L. Lee, J.L. Guan, S. Li, Role of cell surface heparan sulfate proteoglycans in endothelial cell migration and mechanotransduction, J Cell Physiol, 203 (2005) 166-176.

[33] Y. Yao, A. Rabodzey, C.F. Dewey, Jr., Glycocalyx modulates the motility and proliferative response of vascular endothelium to fluid shear stress, Am J Physiol Heart Circ Physiol, 293 (2007) H1023-1030.

[34] Y. Zeng, J.M. Tarbell, The adaptive remodeling of endothelial glycocalyx in response to fluid shear stress, PLoS One, 9 (2014) e86249.

[35] B. Machnicka, A. Czogalla, A. Hryniewicz-Jankowska, D.M. Boguslawska, R. Grochowalska, E. Heger, A.F. Sikorski, Spectrins: a structural platform for stabilization and activation of membrane channels, receptors and transporters, Biochim Biophys Acta, 1838 (2014) 620-634.

[36] P.M. Benz, C. Blume, J. Moebius, C. Oschatz, K. Schuh, A. Sickmann, U. Walter, S.M. Feller, T. Renne, Cytoskeleton assembly at endothelial cell-cell contacts is regulated by alphaII-spectrin-VASP complexes, J Cell Biol, 180 (2008) 205-219.

[37] B. Pontes, P. Monzo, N.C. Gauthier, Membrane tension: A challenging but universal physical parameter in cell biology, Semin Cell Dev Biol, 71 (2017) 30-41.

[38] A. Page-McCaw, A.J. Ewald, Z. Werb, Matrix metalloproteinases and the regulation of tissue remodelling, Nature reviews. Molecular cell biology, 8 (2007) 221-233.

[39] X. Liang, J. Howard, Structural Biology: Piezo Senses Tension through Curvature, Current biology : CB, 28 (2018) R357-R359.

[40] A.P. Christensen, D.P. Corey, TRP channels in mechanosensation: direct or indirect activation?, Nature reviews. Neuroscience, 8 (2007) 510-521.

[41] A. Pietuch, B. Brückner, T. Fine, I. Mey, A. Janshoff, Elastic properties of cells in the context of confluent cell monolayers: Impact of tension and surface area regulation, 2013.

[42] K.M. Giantsos-Adams, A.J. Koo, S. Song, J. Sakai, J. Sankaran, J.H. Shin, G. GarciaCardena, C.F. Dewey, Jr., Heparan Sulfate Regrowth Profiles Under Laminar Shear Flow Following Enzymatic Degradation, Cell Mol Bioeng, 6 (2013) 160-174.

[43] S. Boulant, C. Kural, J.C. Zeeh, F. Ubelmann, T. Kirchhausen, Actin dynamics counteract membrane tension during clathrin-mediated endocytosis, Nature cell biology, 13 (2011) 1124-1131.

[44] P. Miklavc, K. Ehinger, A. Sultan, T. Felder, P. Paul, K.E. Gottschalk, M. Frick, Actin depolymerisation and crosslinking join forces with myosin II to contract actin coats on fused secretory vesicles, J Cell Sci, 128 (2015) 1193-1203. 
Figure legends

a

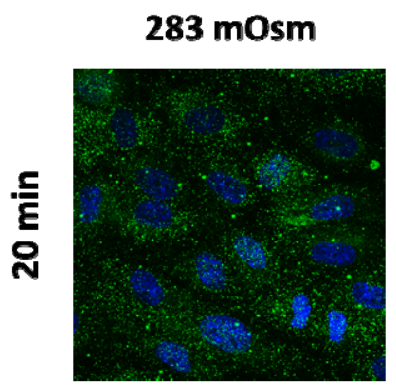

255 mOsm

Syndecan-1/DAPI
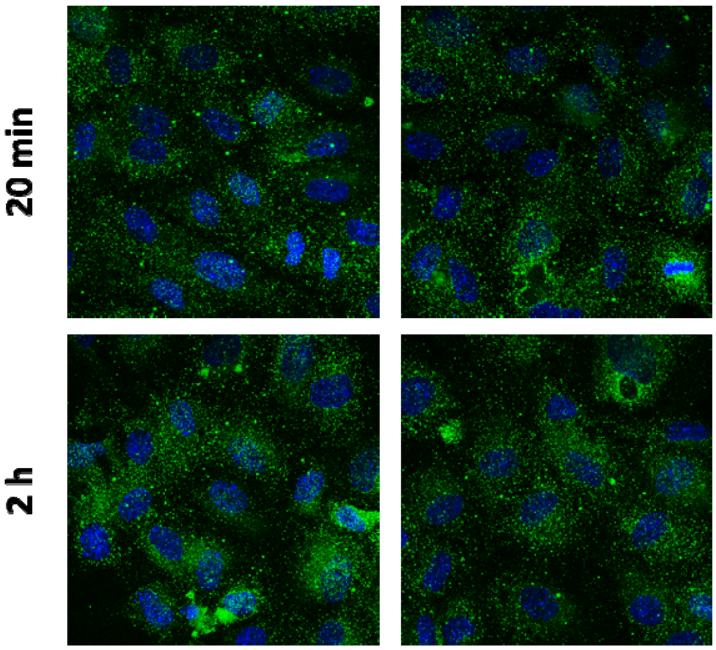

$167 \mathrm{mOsm}$

167 mOsm + Mannitol


b

C

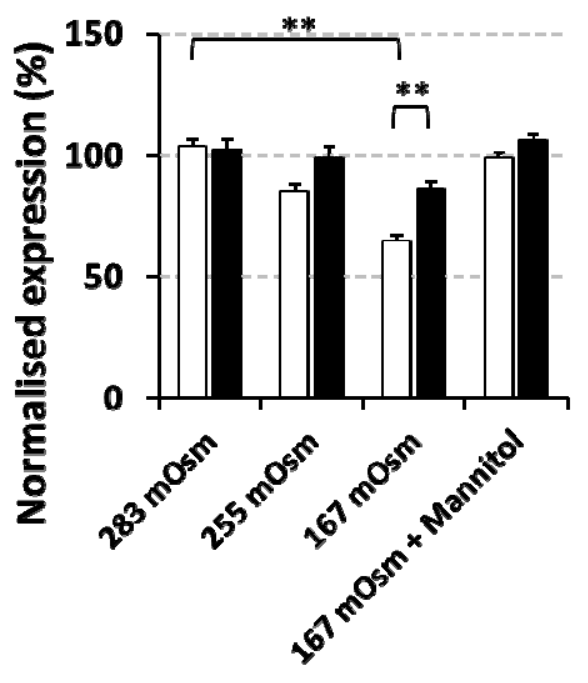

$\square 20 \mathrm{~min} \square \mathbf{2 h}$

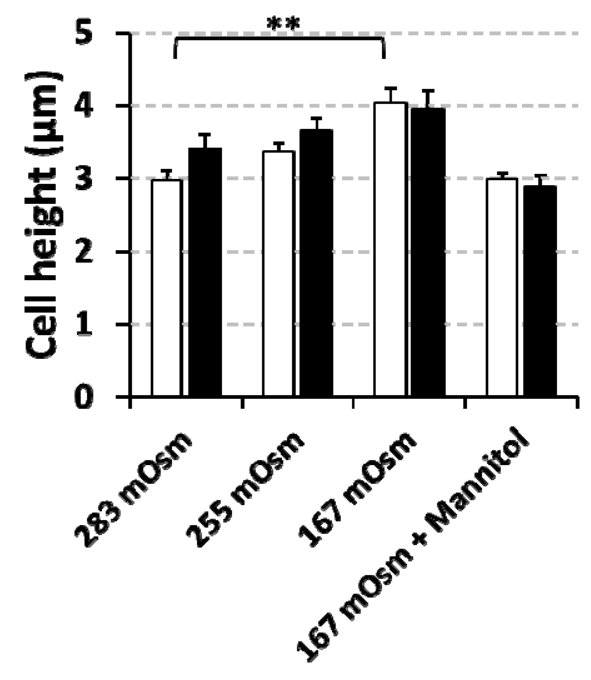

$\square 20 \mathrm{~min} \square 2 \mathrm{~h}$

Fig.1 Increasing membrane tension transiently reduces the expression of syndecan-1. HUVECs were continuously exposed to hypo-osmotic, serum-free medium for $20 \mathrm{~min}$ and $2 \mathrm{~h}$.

a. Confocal images show that the intensity of syndecan-1 is weaken with the decrease of osmolality at $20 \mathrm{~min}$ and subsequently recovered at $2 \mathrm{~h}$. This phenomenon is, however, not observed when the medium osmolality is restored by mannitol to a similar level (320 mOsm) 
as in the pure medium (313 mOsm). Scale bar $=50 \mu \mathrm{m}$. b. Mean fluorescence intensity of syndecan-1 (defined as normalised values to the control i.e. pure medium). ${ }^{* *} P<0.01$ by twoway ANOVA with Turkey from five independent experiments. c. Cell height significantly increases when the medium osmolality is reduced to $167 \mathrm{mOsm}$, suggesting that the cells become swollen and the membrane tension is greatly enhanced. ${ }^{*} P<0.01$ by two-way ANOVA with Turkey from three independent experiments.

a

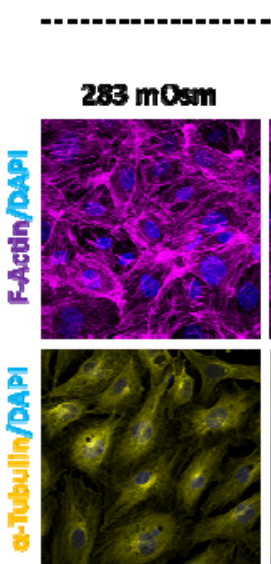

$20 \mathrm{~min}$
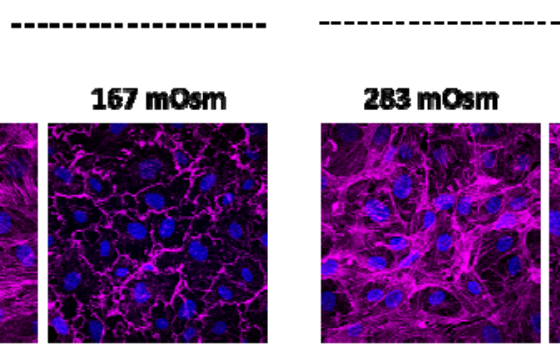

$2 \mathbf{h}$


255 mosm

$167 \mathrm{mosm}$
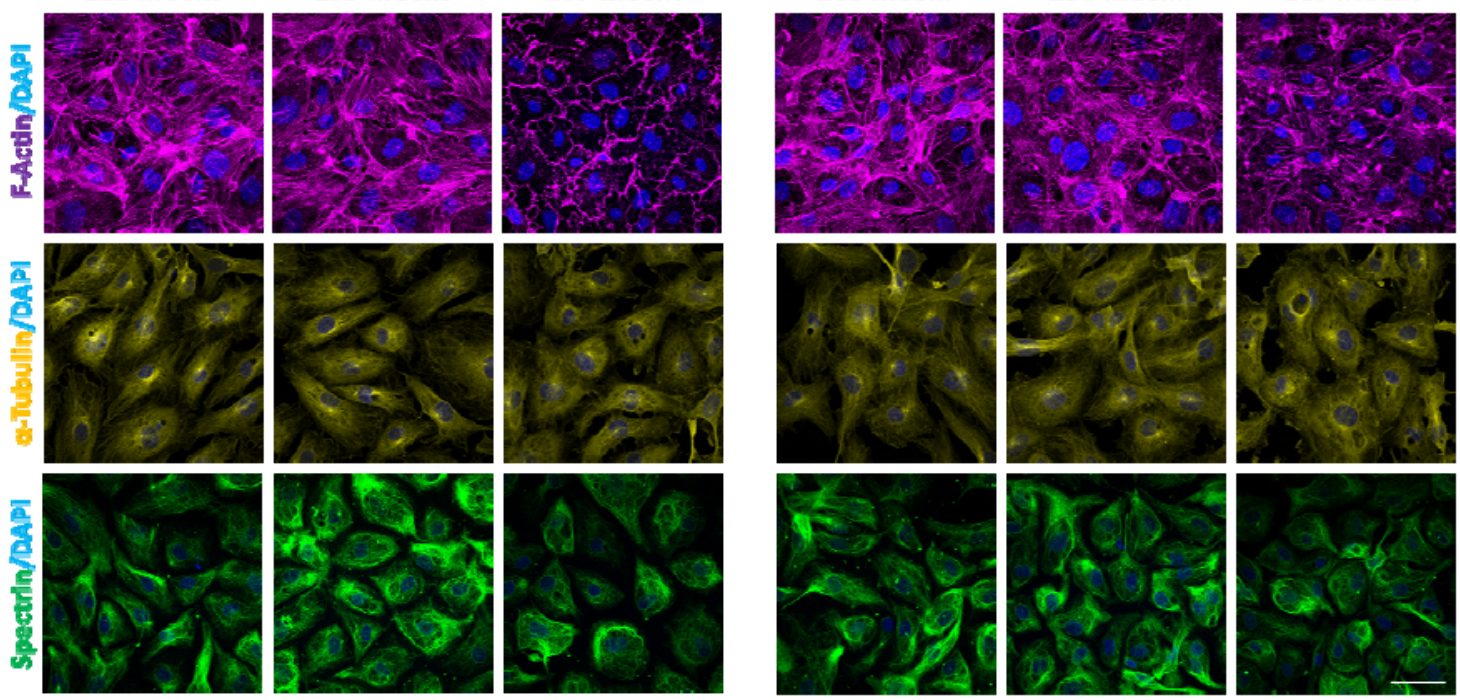

b

c
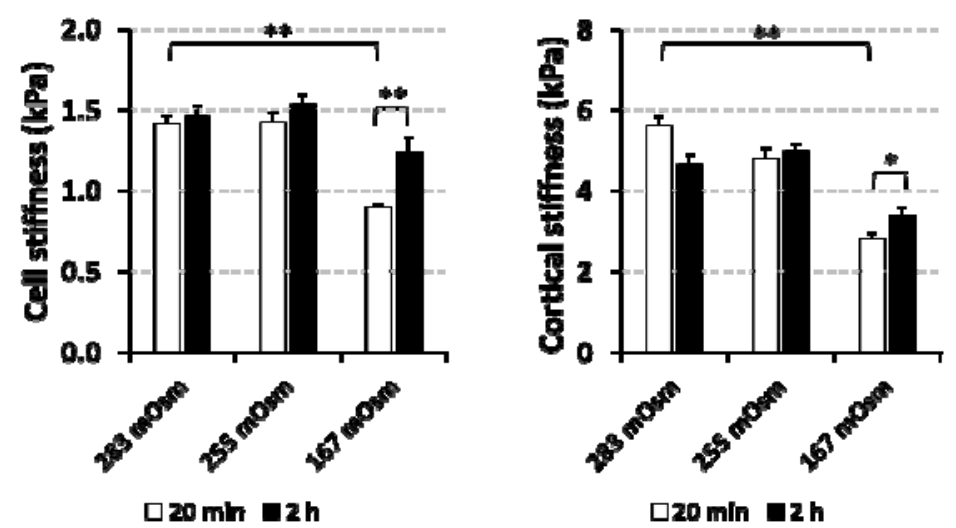

d

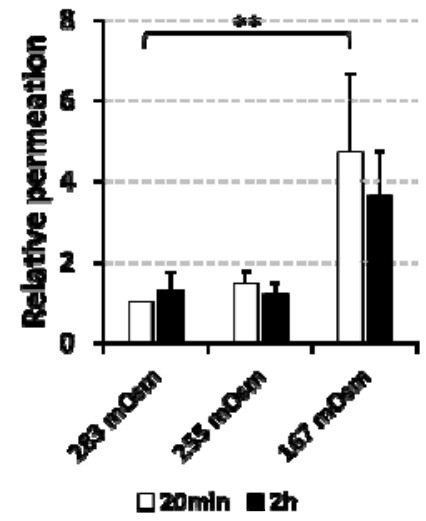




\section{Fig.2 The transient reduction of syndecan-1 is associated with disrupted actin polymerisation.}

Hypo-osmotic stimulated HUVECs were stained for the visualisation of cytoskeletal system.

a. Confocal images show that actin cytoskeleton, rather than microtubule and spectrin, is completely depolymerised following $20 \mathrm{~min}, 167 \mathrm{mOsm}$ hypo-osmotic stress. A partial repolymerisation is observed at the end of $2 \mathrm{~h}$ exposure. Scale bar $=50 \mu \mathrm{m}$. b-d. Changes in cellular stiffness, incl. global cell stiffness (b) and cortical stiffness (c), as well as permeation of FITC-dextran $40 \mathrm{kDa}(\mathrm{d})$, agree with those shown the immunostaining, indicating that the transient reduction of syndecan- 1 is related to the actin reorganisation. ${ }^{*} P<0.05, * * P<0.01$ by two-way ANOVA with Turkey from three independent experiments. 
$20 \mathrm{~min}$

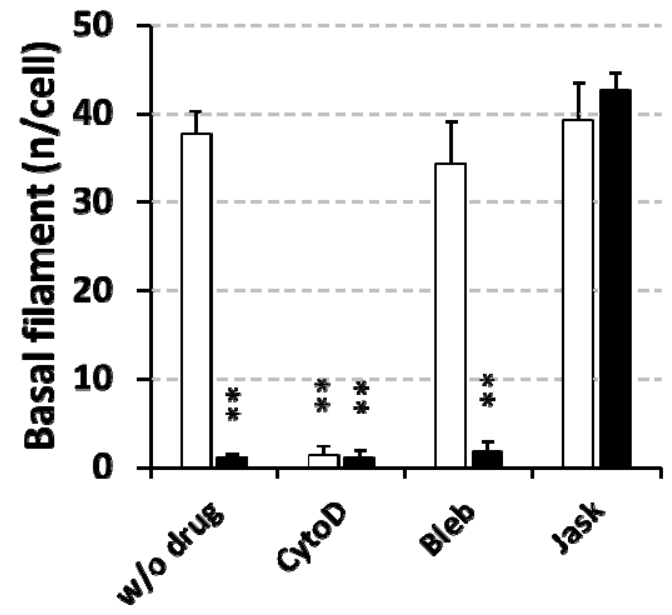

Actomyosin manipulation

$\square 283$ mOsm $\square 167$ mOsm



Actomyosin manipulation

$\square 283$ mOsm 167 mOsm

c

$\mathbf{2 h}$

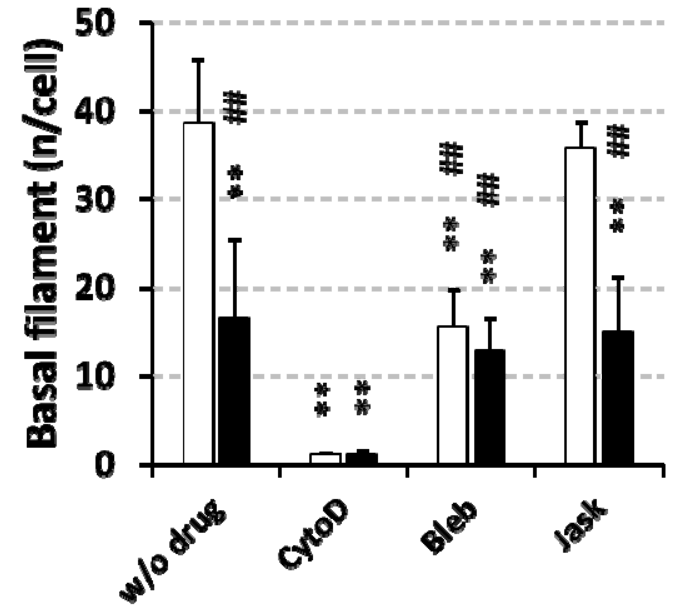

Actomyosin manipulation

$\square 283$ mOsm $\quad 167$ mOsm $\mathbf{2 h}$

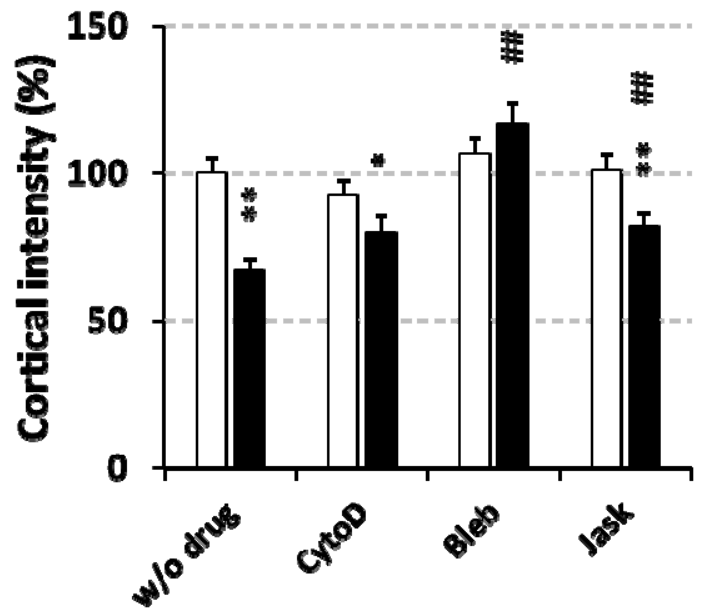

Actomyosin manipulation

$\square 283$ mOsm $\square 167$ mOsm

Fig.3 The transient actin depolymerisation was restored in the presence of jasplakinolide. HUVECs were pre-treated with cytoskeletal drugs for $1 \mathrm{~h}$ and then incubated with hypoosmotic medium concurrent with the same concentration of cytoskeletal drugs for a further 20 $\min (\mathrm{a}, \mathrm{b})$ and $2 \mathrm{~h}(\mathrm{c}, \mathrm{d})$. Cyto D: cytochalasin D $100 \mathrm{nM}$, Bleb: blebbistatin $12.5 \mu \mathrm{M}$, Jask: 
Jasplakinolide $30 \mathrm{nM}$. Actin reorganisation was quantified in terms of basal filament number (a, c) and cortical actin intensity (b, d). The actin-disrupted effect induced by $167 \mathrm{mOsm}$ is sustained in the presence of Cyto D and Bleb, but completely neutralised by Jask at 20 min. Scale bar $=50 \mu \mathrm{m} .{ }^{*} P<0.05,{ }^{* *} P<0.01$ vs. pure medium, \#\# $P<0.01$ between $20 \mathrm{~min}$ and $2 \mathrm{~h}$ by two-way ANOVA with Turkey from three independent experiments.

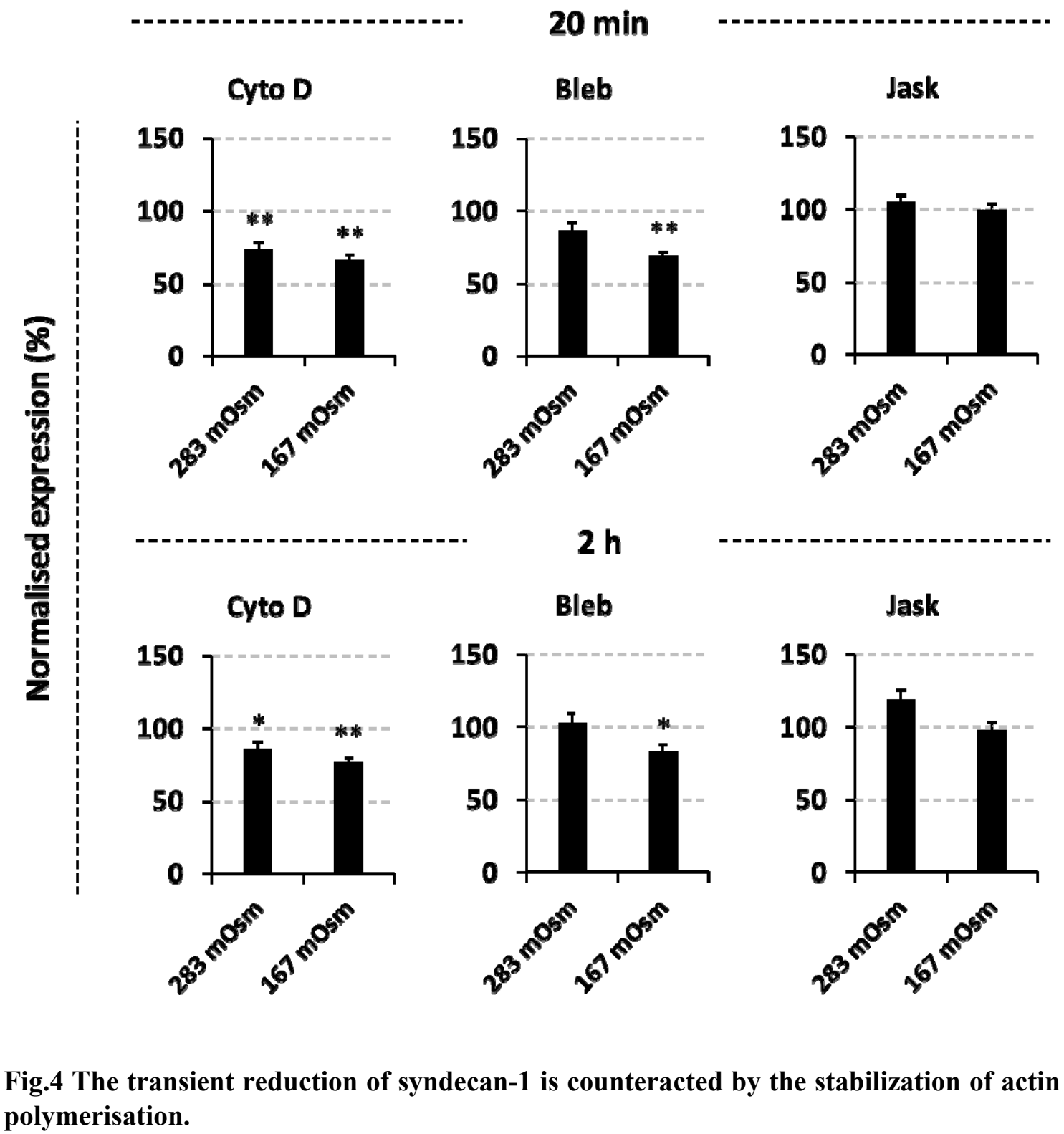


Following actin depolymerisation by cyto $\mathrm{D}$, the reduction of syndecan- 1 expression is sustained at the end of $2 \mathrm{~h}$ stimulation. On the contrary, the transient reduction is completely abrogated with the concurrent incubation of Jask. For those treated with Bleb, the syndecan-1 reduction is not accentuated at $20 \mathrm{~min}$, suggesting that non-muscle myosin II contributes little to the regulation of syndecan- $1 .{ }^{*} P<0.05,{ }^{*} P<0.01$ vs. pure medium by one-way ANOVA with Turkey from five independent experiments.

a

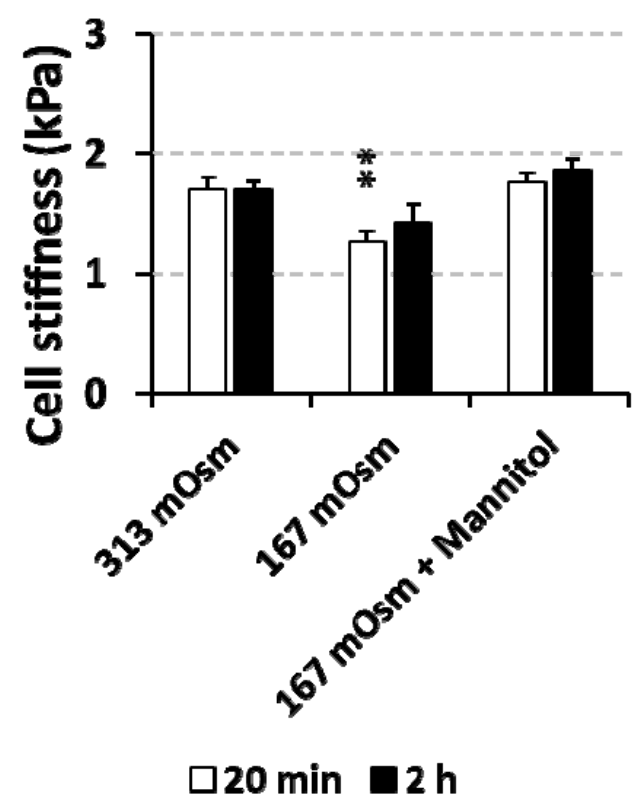

b

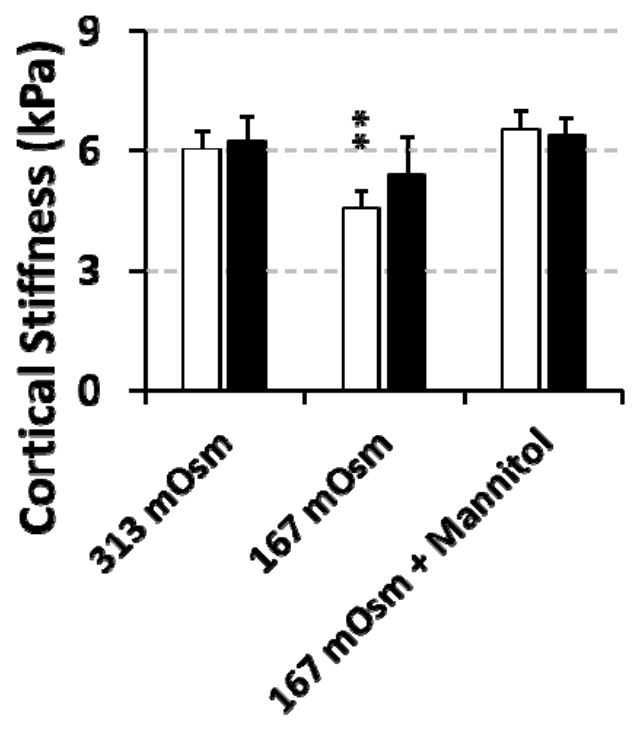

$\square 20 \min \square 2 \mathrm{~h}$

Fig.S1 Measurement of mechanical properties in the absence and presence of mannitol.

Cell softening induced by the hypo-osmotic stress is inhibited by the addition of mannitol. ** $P<0.01$ vs. pure medium by two-way ANOVA with Turkey from three independent experiments. 
a

\section{$\boldsymbol{\alpha}$-Tubulin}

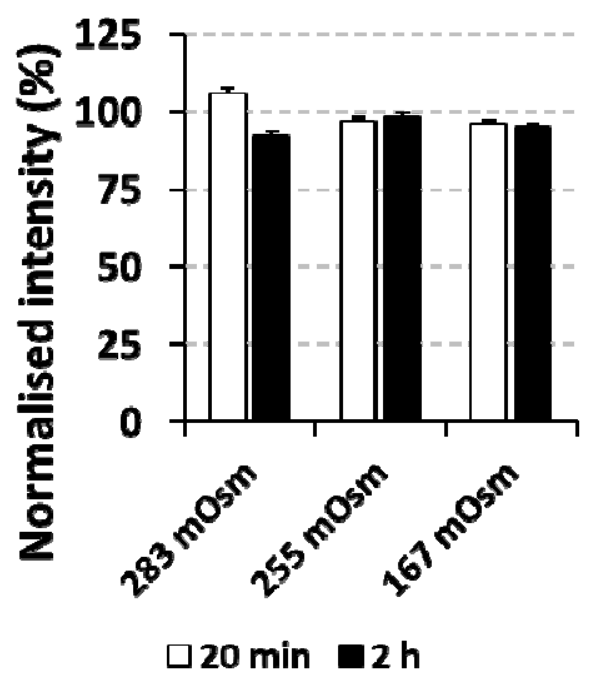

b

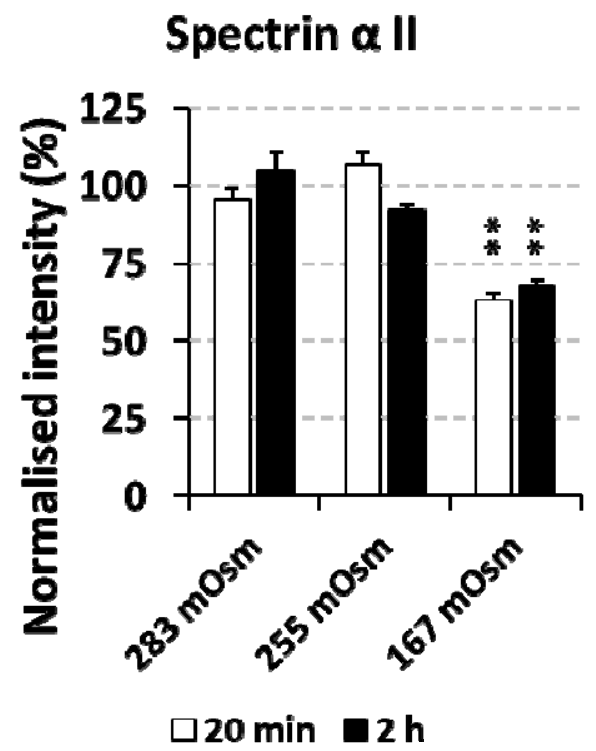

Fig.S2 Measurement of microtubule and spectrin in hypo-osmotically-stressed cells.

Microtubule and spectrin were probed by $\alpha$-tubulin and spectrin $\alpha$ II, respectively, and quantified in the form of mean fluorescence intensity which was normalised to $313 \mathrm{mOsm} . * *$ $P<0.01$ vs. pure medium by two-way ANOVA with Turkey from three independent experiments. 
a
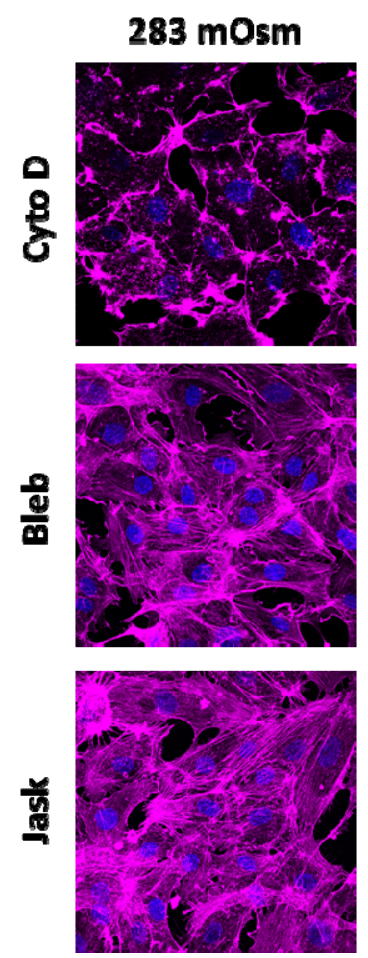

$20 \mathrm{~min}$

167 mOsm


b 2h
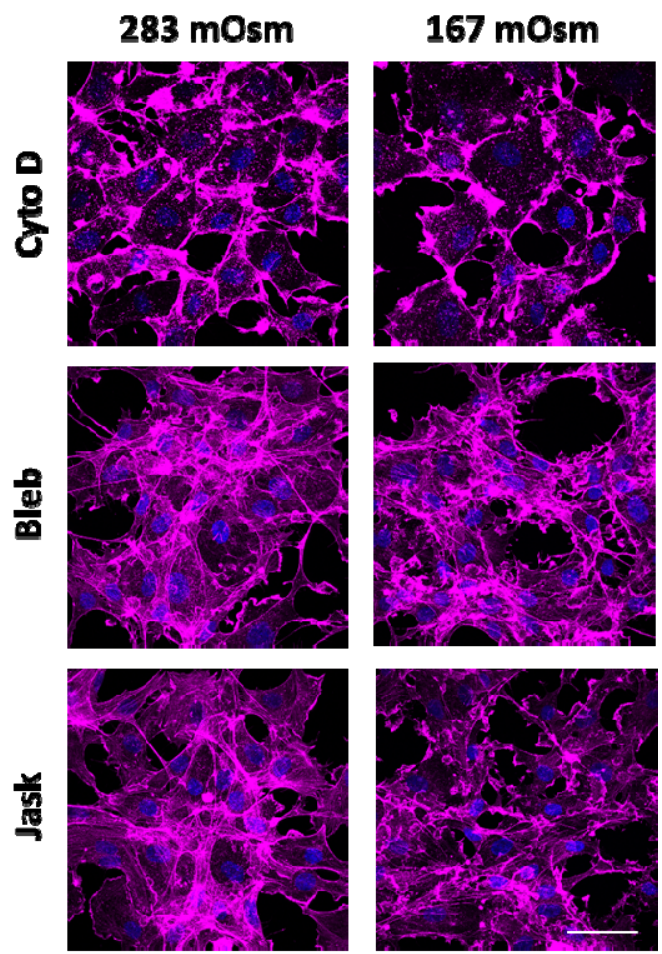

Fig.S3 Representative confocal images showing hypo-osmotic effect on actin cytoskeleton following actin manipulation. Corresponding to Fig. 3. Scale bar $=50 \mu \mathrm{m}$. 
a
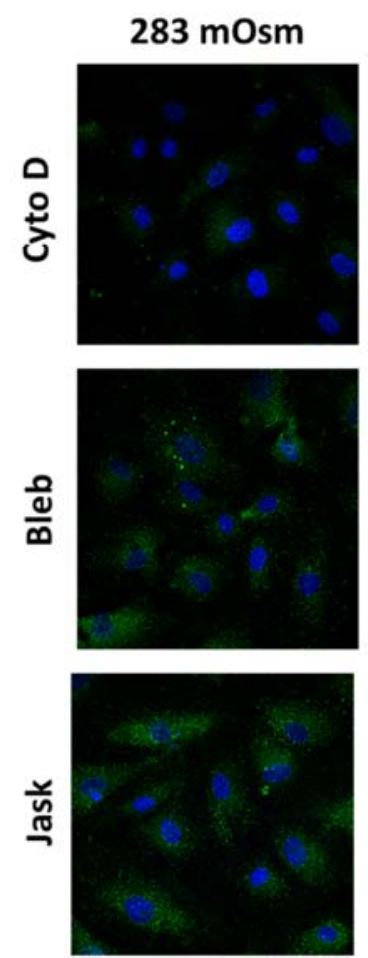

$20 \mathrm{~min}$

167 mOsm
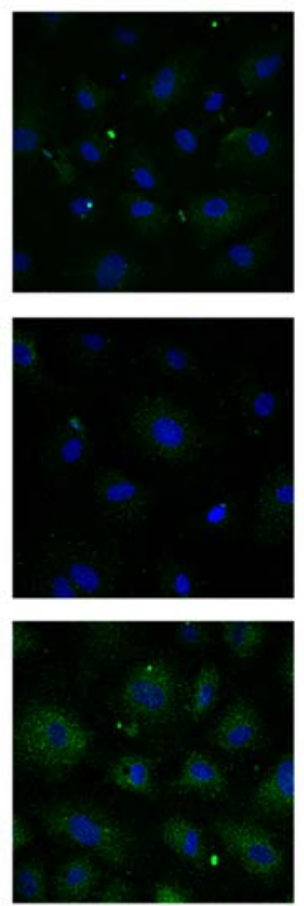

b $2 \mathrm{~h}$

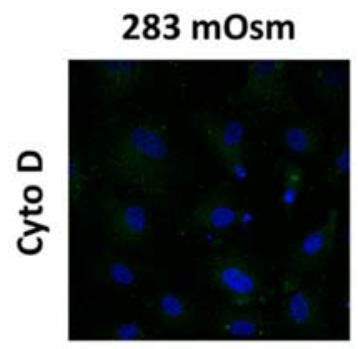

167 mOsm


Fig.S4 Representative confocal images showing hypo-osmotic effect on syndecan-1 expression following actin manipulation. Corresponding to Fig. 4 . Scale bar $=50 \mu \mathrm{m}$. 

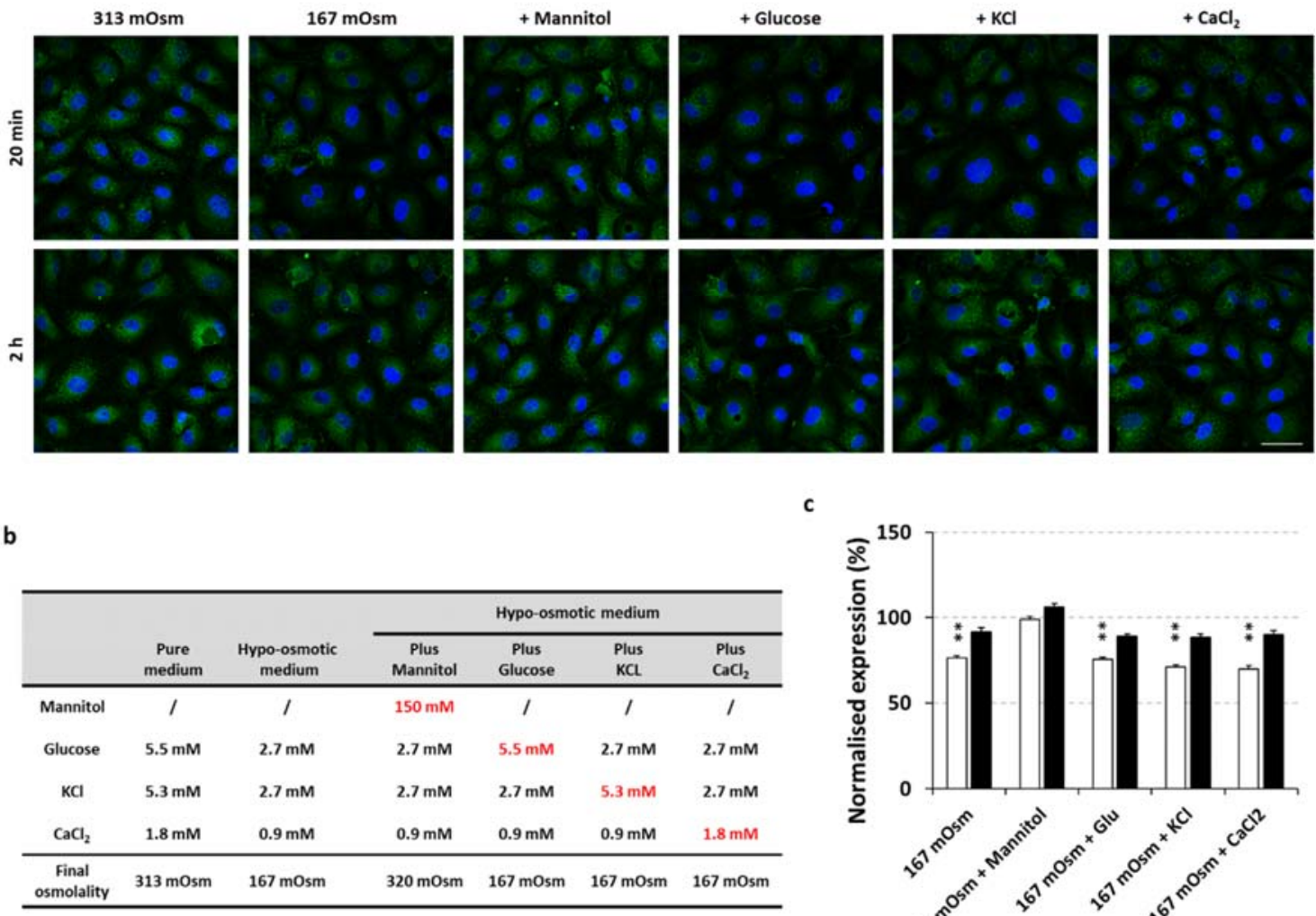

c

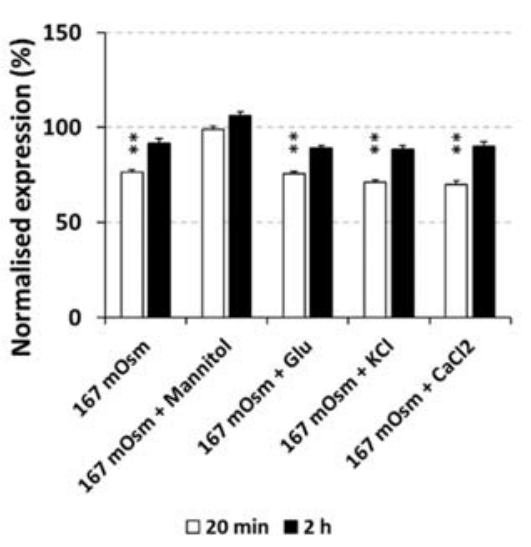

Fig.S5 The effects of ions and glucose on the transient reduction of syndecan-1.

The concentrations of ions and glucose in hypo-osmotic medium (i.e. $167 \mathrm{mOsm}$ ) were restored according to table b. a. representative confocal images showing syndecan-1 staining. Scale bar $=50 \mu \mathrm{m} . \mathrm{b}$. a table summarising the concentrations of additives and the associated medium osmolality. c. The syndecan-1 reduction at $20 \mathrm{~min}$ is abrogated by concurrent incubation with mannitol, where the final osmolality is retrieved to $320 \mathrm{mOsm}$. This phenomenon is not observed if the concentrations of $\mathrm{KCl}, \mathrm{CaCl}_{2}$ and glucose are corrected, respectively, suggesting that it is the altered osmolality that results in the changes of syndecan-1 expression. It should be noted that this experiment does not include $\mathrm{Na}+$ retrieval. This is because $\mathrm{NaCl}$ contributes significantly to the medium osmolality. It is infeasible to alter its concentration without changing the osmolality. ${ }^{* *} P<0.01$ vs. pure medium by two-way ANOVA with Turkey from four independent experiments. 

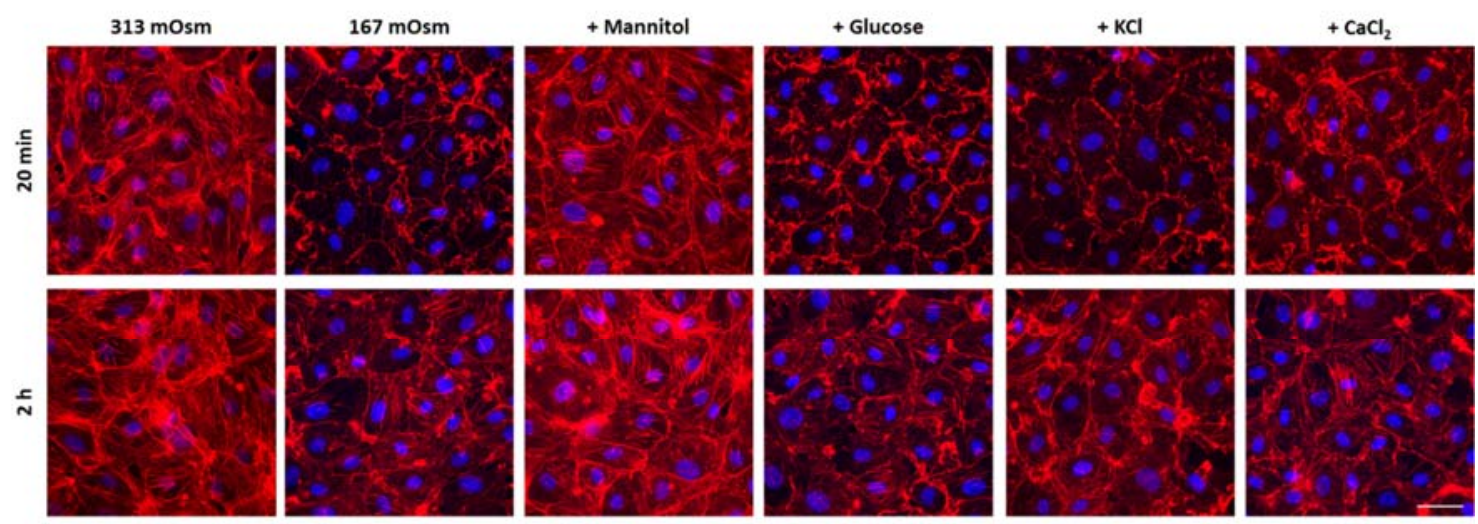

b



c

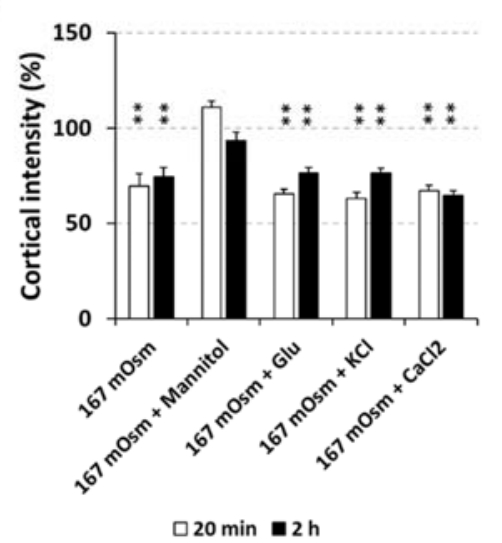

Fig.S6 The effects of ions and glucose on the transient actin depolymerisation.

Consistent with the syndecan-1 expression, actin depolymerisation is suppressed by restoring the medium osmolality with mannitol other than the concentrations of ions and glucose. Scale bar $=50 \mu \mathrm{m} .{ }^{* *} P<0.01$ vs. pure medium, \#\# $P<0.01$ between $20 \mathrm{~min}$ and $2 \mathrm{~h}$ by two-way ANOVA with Turkey from four independent experiments. 